\title{
DIVERSIDAD Y ZOOGEOGRAFÍA DE LOS MOLUSCOS TERRESTRES DE LA SIERRA DE TAMAULIPAS, MÉXICO
}

\author{
DIVERSITY AND ZOOGEOGRAPHY OF THE TERRESTRIAL MOLLUSKS \\ OF THE SIERRA OF TAMAULIPAS, MEXICO
}

\section{Alfonso CORREA-SANDOVAL, ${ }^{1, *}$ RubÉN RODRÍGUEZ-CASTRO, ${ }^{1}$ CrYSTIAN SAdiel VENEGAS-BARRERA, ${ }^{1}$ JORGE VÍCTOR HORTA-VEGA, ${ }^{1}$ LUDIVINA BARRIENTOS-LOZANO ${ }^{1}$ Y JORGE HOMERO RODRÍGUEZ-CASTRO ${ }^{1}$}

\footnotetext{
${ }^{1}$ Instituto Tecnológico de Ciudad Victoria, División de Estudios de Posgrado e Investigación, Boulevard Emilio Portes Gil 1301 Poniente, Apdo. Postal 175, C. P. 87010, Ciudad Victoria, Tamaulipas, México.

* Autor de correspondencia: <alf_correas@hotmail.com>
}

Recibido: 29/06/2016; aceptado: 12/12/2016

Editor responsable: Pedro Reyes Castillo

Correa-Sandoval, A., Rodríguez-Castro, R., Venegas-Barrera, C. S., Horta-Vega, J. V., Barrientos-Lozano, L. y RodríguezCastro, J. H. (2017) Diversidad y zoogeografía de los moluscos terrestres de la sierra de Tamaulipas, México. Acta Zoológica Mexicana (n.s.), 33(1), 76-88.

RESUMEN. La diversidad y biogeografía de los moluscos terrestres mexicanos es pobremente conocida. Entre octubre de 1988 y noviembre de 2005 fueron estudiados los gastrópodos terrestres de la Sierra de Tamaulipas, obteniéndose 482 muestras de 30 localidades en diferentes tipos de vegetación. Se registraron 31 géneros y 46 especies pertenecientes a 18 familias. La diversidad malacológica es mayor a la de otras áreas naturales de importancia para la conservación en México. Las principales afinidades zoogeográficas son la neotropical principalmente y neártica de 17 especies (37\% de la malacofauna) y el endemismo de 11 especies (24\%). La familia con mayor número de especies endémicas es Spiraxidae con seis. El valor biogeográfico promedio es de 3.78 .

Palabras clave: gastrópodos terrestres, taxonomía, biogeografía, endemismo, Sierra de Tamaulipas.

\section{INTRODUCCIÓN}

El noreste de México es la región del país en la que más localidades se han visitado para el trabajo de campo en relación a moluscos terrestres (Correa-Sandoval 2003, Correa-Sandoval \& Salazar-Rodríguez 2005, Correa-Sandoval et al. 2012). Sin embargo, existen áreas geográficas como la Sierra de Tamaulipas, que requieren un mayor análisis al ser, por su extensión y posición geográfica, uno de los principales componentes montañosos aislados de la Sierra Madre Oriental.
Correa-Sandoval, A., Rodríguez-Castro, R., Venegas-Barrera, C. S., Horta-Vega, J. V., Barrientos-Lozano, L., \& RodríguezCastro, J. H. (2017) Diversity and Zoogeography of terrestrial mollusks in Sierra of Tamaulipas, Mexico. Acta Zoológica Mexicana (n.s.), 33(1), 76-88.

ABSTRACT. Diversity and biogeography of Mexican terrestrial gastropods is poorly known. The terrestrial mollusks of the Sierra of Tamaulipas were surveyed from October 1988 to November 2005. A total of 482 samples were obtained from 30 localities, characterized with different vegetation types. Thirty-one genera and 46 species belonging to 18 families are recorded. The primary zoogeographical relationships are characterized by the presence of neotropical and neartic affinities (17 species: 37\%) and the endemic taxa (11 species: $24 \%$ ). The family Spiraxidae has the largest number of endemic species (6). The biogeographical value in this area is 3.78 .

Key words: Terrestrial gastropods, taxonomy, biogeography, endemism, Sierra of Tamaulipas.

La mayor parte de los registros de moluscos terrestres en Tamaulipas, como es común a diversos grupos faunísticos y florísticos, pertenecen a la Sierra Madre Oriental. Sólo a partir de 1987, con la creación de la Colección de Moluscos del Instituto Tecnológico de Ciudad Victoria, se ha revisado con mayor regularidad la Sierra de Tamaulipas.

Hasta ahora sólo tres especies nuevas de gastrópodos terrestres han sido descritas específicamente para esta área geográfica o bien para áreas cercanas. En 1936 Pilsbry y Vanatta describieron a Guillarmodia pygmaea y Euglan- 
dina jacksoni para el municipio de González, ubicado al sur de la Sierra de Tamaulipas; y en 1994 Thompson y Correa-Sandoval describieron a Coelocentrum torosum para el noroeste de la localidad de El Pirulí, en el norte de la Sierra de Tamaulipas.

Este estudio establece la composición taxonómica de la malacofauna terrestre de la Sierra de Tamaulipas, la proporción de sus afinidades biogeográficas y las analiza comparativamente en relación a otras áreas geográficas, tanto de Tamaulipas como de otros estados de la República Mexicana. También revisa la distribución geográfica potencial de las especies para identificar las zonas con mayor riqueza de moluscos.

\section{MATERIALES Y MÉTODOS}

Se visitaron 30 localidades (Apéndice 1) para realizar los muestreos y colectas directas de acuerdo a las recomendaciones de Coney et al. (1981) y Correa-Sandoval et al. (1998). El trabajo de campo se realizó en función del tipo de vegetación como el hábitat primario de la malacofauna terrestre (Pérez et al. 1996, Tattersfield et al. 2001).

Se revisó por su accesibilidad la Sierra de Tamaulipas en un eje transversal oriente-poniente, así como diversos sitios con tipos de vegetación diferente (bosque de pino en el área central de la Sierra de Tamaulipas) a los hallados comúnmente en el área de estudio (matorral submontano, bosque tropical caducifolio, bosque tropical subcaducifolio, bosque de encino y pastizal).

Las muestras del suelo se tamizaron y revisaron en laboratorio de acuerdo a Cameron y Redfern (1976) y Correa-Sandoval et al. (1998). Los animales ya relajados se conservaron en alcohol etílico al 70\% (Solem et al. 1980). Se revisaron un total de 482 lotes (3121 ejemplares).

El material determinado y etiquetado está depositado en las Colecciones Malacológicas del Instituto Tecnológico de Ciudad Victoria (TAM-CEVS-CC-0001-15) y el Museo de Historia Natural de Florida, Universidad de Florida.

El orden de las familias (Apéndice 2) sigue la propuesta de Bouchet \& Rocroi (2005) la cual es seguida por Thompson (2011) en la revisión de los moluscos terrestres de México y Centroamérica. Los géneros y especies se ordenaron alfabéticamente. La caracterización zoogeográfica en el continente americano es a nivel específico o subespecífico según el caso y de acuerdo a Correa-Sandoval (1993, 1999, 2003), criterio seguido con algunas modificaciones por Rangel-Ruiz et al. (2004). El índice biogeográfico resulta del promedio de los valores asignados en orden decreciente según la afinidad malacogeográfica de cada especie de molusco terrestre a partir del endemismo y hasta la distribución más amplia o panamericana.

Las áreas con mayor riqueza de especies en el área de estudio se establecieron con base a los modelos predictivos de distribución potencial (MPDP), los cuales son métodos computarizados que permiten estimar la probabilidad de que una especie esté presente, dadas las condiciones ambientales en el área de estudio (Guisan \& Zimmermann 2000, Hirzel \& Lay 2008). El supuesto de estos análisis es que la especie sólo ocurrirá en aquellas condiciones ambientales en donde se satisfagan sus requerimientos de nicho (Soberon \& Peterson 2005). Los MPDP suponen que se puede predecir la ocurrencia de la especie en los sitios donde no ha sido observada a partir de los registros de colecta, variables ambientales y un algoritmo de predicción (Richards et al 2007). En el presente estudio, la predicción de la distribución potencial se realizó en el paquete Maxent ver 3.2. (Phillips et al. 2006), debido a que es uno de los algoritmos más robustos para la predicción de la distribución de las especies (Elith et al. 2006). El algoritmo busca la combinación de variables con máxima entropía, es decir, la más cercana a la distribución uniforme de los sitios en donde fue registrada la especie.

El algoritmo de MaxEnt generó un modelo de distribución que utiliza datos de sólo presencia, donde los píxeles del área de estudio constituyen el espacio en el cual se define la probabilidad de distribución de máxima entropía (Phillips et al. 2006). El valor de umbral de convergencia, para encontrar la distribución más cercana a la distribución empírica, fue de 0.00001, tuvo un máximo de 1000 iteraciones. En la generación del modelo de distribución potencial, se utilizaron los cuatro tipos de características: el producto bruto (lineal), cuadrado (cuadrático), producto de las variables ambientales y las características binarias derivadas del umbral de variables ambientales (función de umbral). Del modelo generado se calculó el área bajo la curva (AUC) para estimar la probabilidad de que el modelo generado fue mejor que un modelo generado al azar. Una clasificación perfecta tiene un AUC = 1.0.

La predicción de la distribución geográfica se realizó utilizando variables que representan el clima, elevación sobre el nivel medio del mar y la vegetación. La resolución espacial de las capas es de aproximadamente 9 $\mathrm{km}^{2}$. Las variables climáticas y elevación se obtuvieron de WorldClim (www.worldclim.org/) y las variables de cobertura vegetal de Global Land Cover Facility (http:// 
www.landcover.org/index.shtml). También se obtuvo el índice de aridez (http://csi.cgiar.org/aridity/index.asp). Las capas temáticas se generaron con los programas ArcGis (ver 10.0 ESRI, 2009) e Idrisi Taiga (Clarck Lab).

Los modelos generados fueron realizados sólo en las especies que tuvieron ocho registros. En caso de que tuvieran un número menor, se generó el modelo para el área de estudio. Los registros de colecta fueron obtenidos de artículos, tesis, reportes y de diversas bases de datos nacionales e internacionales (CONABIO y GBIF). Los registros fueron refinados, para lo cual se eliminaron aquellos registros donde no se cuente con al menos el nombre de la localidad o este se encuentre fuera de la distribución conocida de la especie (más de $50 \mathrm{~km}$ ). A todos los registros se les obtuvieron las coordenadas geográficas a partir de la literatura y con el programa en línea Google Earth, GEOlocate (ver 2.03) y mapas topográficos (escala 1: 50,000).

\section{RESULTADOS Y DISCUSIÓN}

Las 46 especies y 31 géneros de moluscos terrestres de la Sierra de Tamaulipas se distribuyen en 18 familias (Apéndice 2). Las familias más numerosas en especies son Spiraxidae con 10 y Helicinidae, Orthalicidae y Polygyridae todas con siete especies.

Las especies con mayor distribución son PraticoleIla berlandieriana y Helicina chrysocheila chrysocheila distribuidas en 21 y 20 localidades respectivamente, seguidas de Euglandina texasiana texasiana presente en 14 localidades.

Las localidades con mayor número de especies son km 68 Carretera Victoria - Soto La Marina; Rancho La Palma, Carretera Victoria - Soto La Marina (lado izquierdo de la carretera); Carretera La Pesca - Soto La Marina (después de Vista Hermosa), todas con 24 especies; le siguen km 64.5 Carretera Victoria - Soto La Marina (des- pués de El Pirulí) y Carretera La Pesca - Soto La Marina (antes de la desviación al Capote), con 20 y 21 especies respectivamente. En estas localidades se presenta principalmente vegetación de bosque tropical subcaducifolio o caducifolio.

La Sierra de Tamaulipas presenta un número comparativamente mayor (a veces más del doble) de especies de moluscos terrestres con respecto a otras áreas naturales de importancia para la conservación en México (Cuadro 1), lo cual resalta en parte su importancia faunística. Sin embargo, deben considerarse como factores para explicar estas diferencias la intensidad del trabajo de campo realizado, los métodos empleados para el muestreo o colecta, además de parámetros ecológicos como el tipo de vegetación, entre otros.

El 63 y 46\% del total de géneros y especies respectivamente del estado se presentan en la Sierra de Tamaulipas. El 78\% (36 especies) de la fauna de moluscos terrestres del área de estudio se comparte con la Sierra Madre Oriental en Tamaulipas.

El principal componente zoogeográfico de la malacofauna de la Sierra de Tamaulipas es el neotropical principalmente y neártico (37\%: 17 especies), seguido del endemismo del 24\% (11 especies).

Similar orden en estas mismas afinidades se presenta en el Área Natural Protegida de Altas Cumbres con el 42\% (18 especies) y 21\% (9 especies), respectivamente (Martínez- Ramírez \& Rodríguez-Castro 1997; CorreaSandoval et al. 2012). Una posición invertida de estas mismas afinidades se presenta en la Reserva de la Biosfera El Cielo, con el endemismo del 34\% (15 especies) y el carácter neotropical principalmente y neártico del 32\% (14 especies) según Correa-Sandoval \& Rodríguez-Castro (2005). Igual sucede en general en el Sur de Tamaulipas con el 43\% (45 especies) de endemismo y 23\% (24 especies) del componente neotropical principalmente y neártico (Correa-Sandoval et al. 2012), (Cuadro 2, Figs. 1, 2 y 3).

Cuadro 1. Comparativo del número de especies de moluscos terrestres de la Sierra de Tamaulipas y algunas otras áreas de importancia para la conservación en México.

\begin{tabular}{ccccc}
\hline Sierra de Tamaulipas & $\begin{array}{c}\text { Reserva El Cielo } \\
\text { (Tamaulipas) }^{1}\end{array}$ & $\begin{array}{c}\text { Parque Estatal La Sierra } \\
\text { (Tabasco) }^{2}\end{array}$ & $\begin{array}{c}\text { Parque Estatal Agua } \\
\text { Blanca (Tabasco) }^{3}\end{array}$ & $\begin{array}{c}\text { Área Focal de Ixcan } \\
(\text { Chiapas) }\end{array}$ \\
\hline 46 & 44 & 20 & 22 & 29 \\
\hline
\end{tabular}

${ }^{1}$ Correa-Sandoval \& Rodríguez-Castro (2005)

${ }^{2}$ Rangel- Ruiz \& Gamboa-Aguilar (1998, 2001).

${ }^{3}$ Rangel - Ruiz et al. (2004);

${ }^{4}$ Avendaño (2004).

${ }^{5}$ Avendaño et al. (2010). 
Cuadro 2. Número de especies de moluscos terrestres y porcentaje comparativo de los componentes zoogeográficos de diversas áreas geográficas en el Estado de Tamaulipas. Simbología: El endemismo o distribución especial para Tamaulipas, Nuevo León o San Luis Potosí se simboliza "EN", la afinidad neotropical "NT", la afinidad neártica "NA", la principalmente neotropical y alguna presencia en el neártico "NTp y NA”, la principalmente neártica y alguna presencia en el neotrópico “NAp y NT” y la distribución panamericana "PA”, la cual ocurre al norte y sur del Trópico de Cáncer.

\begin{tabular}{|c|c|c|c|c|c|c|c|c|}
\hline \multirow[t]{2}{*}{ Afinidad } & \multicolumn{2}{|c|}{ Sierra de Tamaulipas } & \multicolumn{2}{|c|}{$\begin{array}{l}\text { Área natural protegida } \\
\text { Altas Cumbres }^{1}\end{array}$} & \multicolumn{2}{|c|}{$\begin{array}{c}\text { Reserva de la biosfera } \\
\text { El Cielo }{ }^{2}\end{array}$} & \multicolumn{2}{|c|}{ Sur de Tamaulipas } \\
\hline & Especies & $\%$ & Especies & $\%$ & Especies & $\%$ & Especies & $\%$ \\
\hline NAp y NT & 2 & 4 & 3 & 7 & 1 & 2 & 7 & 7 \\
\hline NT & 9 & 20 & 4 & 12 & 11 & 27 & 14 & 13 \\
\hline NA & 0 & 0 & 0 & 0 & 0 & 0 & 1 & 1 \\
\hline Total especies & \multicolumn{2}{|c|}{46} & \multicolumn{2}{|c|}{42} & \multicolumn{2}{|c|}{43} & \multicolumn{2}{|c|}{105} \\
\hline
\end{tabular}

${ }^{1}$ Martínez- Ramírez \& Rodríguez-Castro (1997)

${ }^{2}$ Correa-Sandoval \& Rodríguez-Castro (2005)

${ }^{3}$ Correa-Sandoval et al. (2012)

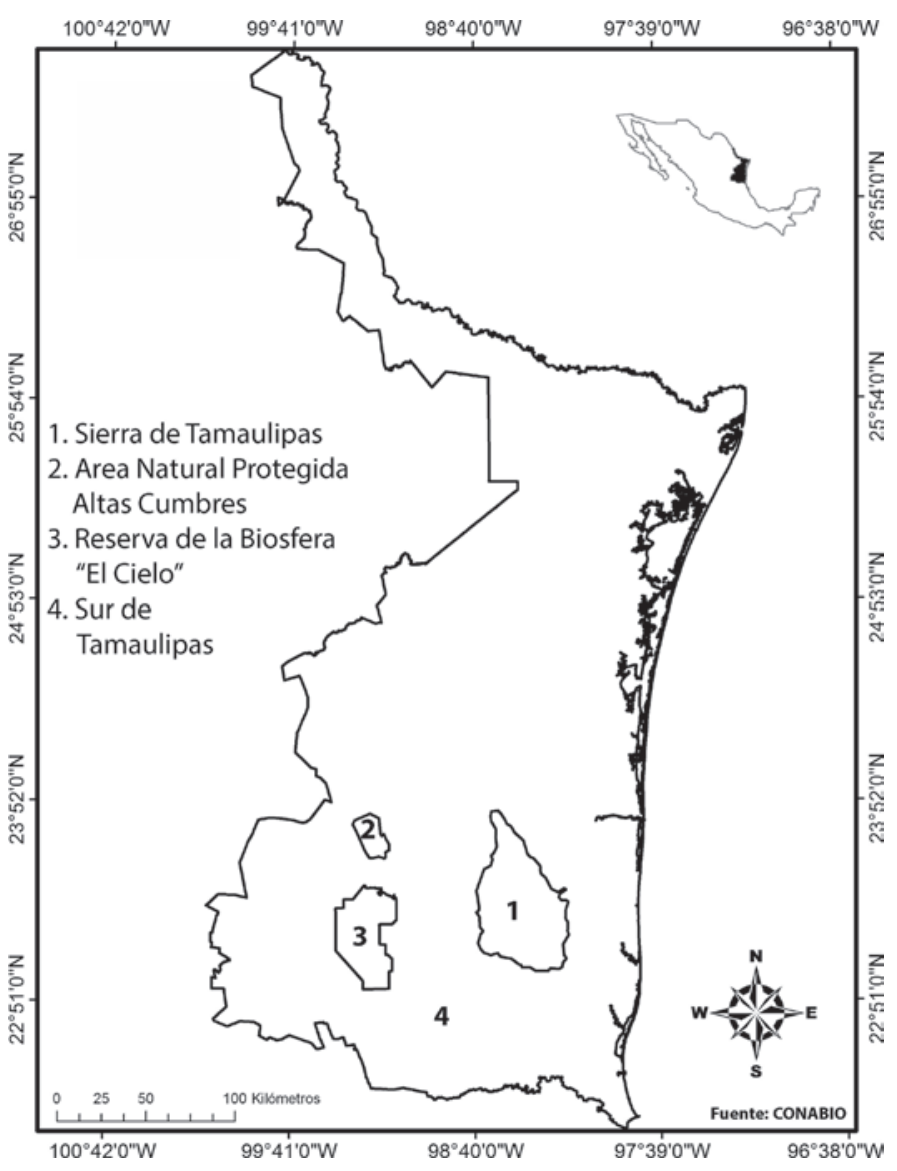

Figura 1. Ubicación de la Sierra de Tamaulipas y otras áreas geográficas en el estado de Tamaulipas.
Al comparar las afinidades de los moluscos terrestres de la Sierra de Tamaulipas con otras zonas del noreste de México (Cuadro 3, Figs. 4 y 5) el carácter neotropical principalmente y neártico es el segundo en importancia en el sur de Nuevo León (32\%: 27 especies). Aquí el endemismo es del 40\% (34 especies) siendo el más importante, al igual que en el oriente de San Luis Potosí donde este componente es del 32\% (28 especies), (Correa-Sandoval 1999, Correa-Sandoval et al. 2007). En el norte de Veracruz el carácter exclusivamente neotropical se halla en el $39 \%$ de la malacofauna terrestre según Correa-Sandoval (2000), y en la Sierra de Tamaulipas es del 20\% (9 especies).

Lo anterior indica que la parte media oriental del estado de Tamaulipas en la cual se halla ubicada la Sierra de Tamaulipas es una zona de transición zoogeográfica y en particular de las provincias malacológicas terrestres Texana, de la Sierra Madre Oriental y Veracruzana.

Entre las familias con especies endémicas en el área de estudio destaca Spiraxidae con el 54\% (6 especies) del total malacofaunístico (Cuadro 4), siendo este un valor más elevado al hallado para toda la zona centro y sur de Tamaulipas (48\%) en esta misma familia (Correa-Sandoval et al. 2012). Un valor aún más elevado (57\%) se presenta en la región oriental de San Luis Potosí (CorreaSandoval 1999).

La familia Spiraxidae es la que mostró más endemismos (6 especies: 60\% en relación con el número total de especies de la familia en el área de estudio), seguida por 


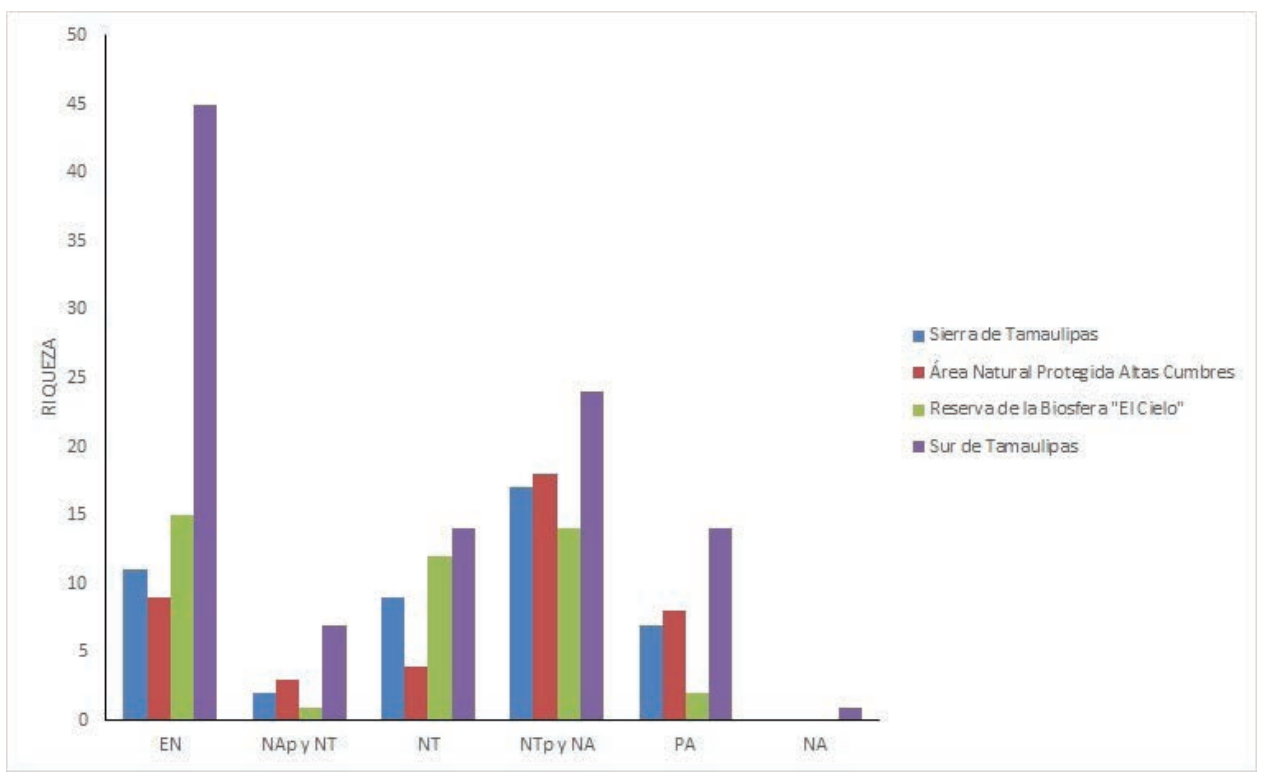

Figura 2. Comparativo de componentes zoogeográficos por número de especies de moluscos terrestres en diferentes áreas de Tamaulipas. Simbología del Cuadro 2.

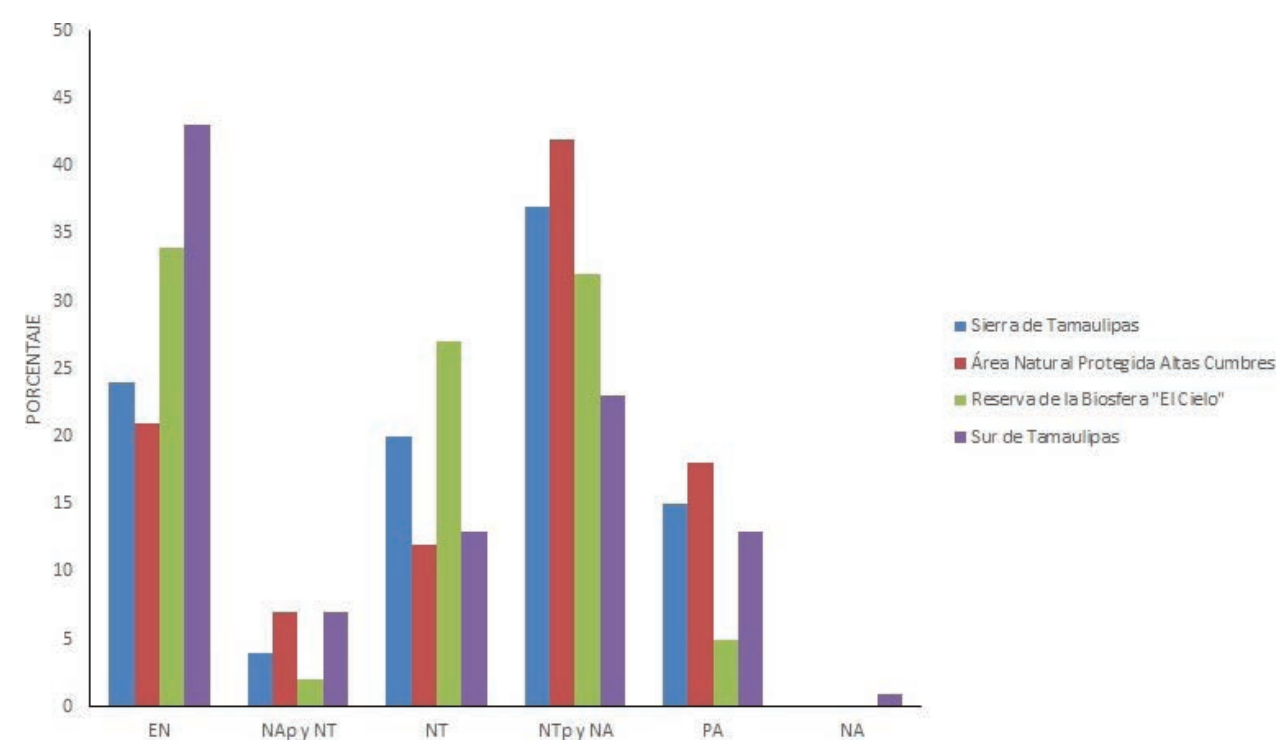

Figura 3. Porcentaje comparativo de componentes zoogeográficos de los gastrópodos terrestres en diversas áreas de Tamaulipas. Simbología del Cuadro 2.

la familia Polygyrydae con dos especies (33\%). Las familias Helicinidae, Zonitidae y Eucalodiidae presentaron un endemismo cada una (Cuadro 4).

Del total de 11 especies caracterizadas como endémicas en este estudio, seis lo son de diversas zonas en la región noreste de México (Schasicheyla fragilis, Guillarmodia dalli, G. victoriana, Salasiella hinkleyi, Patulopsis montereyensis victorianus y Polygyra ariadnae). Las restantes lo son específicamente de la Sierra de Tamaulipas y áreas adyacentes (Coelocentrum torosum, Euglandina jacksoni, Euglandina sp. nov., Spiraxis sp. nov. y Polygyra sp. nov.).

Otras especies presentan particularidades en su distribución geográfica regional: Polygyra implicata se halla 
Cuadro 3. Número de especies y porcentaje comparativo de componentes zoogeográficos de los moluscos terrestres de la Sierra de Tamaulipas y otras áreas del noreste de México. Simbología del Cuadro 2.

\begin{tabular}{|c|c|c|c|c|c|c|c|c|}
\hline \multirow[t]{2}{*}{ Afinidad } & \multicolumn{2}{|c|}{ Sierra de Tamaulipas } & \multicolumn{2}{|c|}{ Sur de Nuevo León ${ }^{1}$} & \multicolumn{2}{|c|}{ Oriente de San Luis Potosí ${ }^{2}$} & \multicolumn{2}{|c|}{ Norte de Veracruz ${ }^{3}$} \\
\hline & Especies & $\%$ & Especies & $\%$ & Especies & $\%$ & Especies & $\%$ \\
\hline EN & 11 & 24 & 34 & 40 & 28 & 32 & 14 & 27 \\
\hline NAp y NT & 2 & 4 & 5 & 6 & 8 & 9 & 2 & 4 \\
\hline NT & 9 & 20 & 0 & 0 & 24 & 28 & 20 & 39 \\
\hline NTp y NA & 17 & 37 & 27 & 32 & 15 & 17 & 11 & 22 \\
\hline PA & 7 & 15 & 13 & 15 & 11 & 13 & 4 & 8 \\
\hline NA & 0 & 0 & 5 & 6 & 1 & 1 & 0 & 0 \\
\hline Total especies & \multicolumn{2}{|c|}{46} & \multicolumn{2}{|c|}{84} & \multicolumn{2}{|c|}{87} & \multicolumn{2}{|c|}{51} \\
\hline
\end{tabular}

${ }^{1}$ Correa-Sandoval et al. (2007)

${ }^{2}$ Correa-Sandoval (1999)

${ }^{3}$ Correa-Sandoval (2000)

principalmente en la Sierra de Tamaulipas y áreas cercanas, pero se presenta también en zonas bajas orientales de la Sierra Madre Oriental; y Practicolella griseola cuya única localidad conocida documentada en Tamaulipas cercana a la Sierra Madre Oriental es El Nacimiento, en el municipio de El Mante (Correa-Sandoval et al. 2012).

La presencia de Thysanophora fuscula (especie con distribución disyunta: Jamaica, Chiapas, Tamaulipas) señalada en el estado por Pilsbry (1920) en Tampico y Cd. Victoria, se confirma por varias localidades mencionadas por Correa-Sandoval et al. (2012) además de las de este estudio en la Sierra de Tamaulipas.
En un comparativo del índice biogeográfico de la malacofauna terrestre de la Sierra de Tamaulipas con el de la región oriental de San Luis Potosí (Correa-Sandoval et al. 2009), limitado a los tipos de vegetación compartidos (bosque de encino, bosque tropical subcaducifolio y pastizal), resulta mayor en ésta última zona (Cuadro 5). El valor medio del índice biogeográfico de la fauna de moluscos terrestres para todos los tipos de vegetación en la Sierra de Tamaulipas es de 3.78.

Es necesario realizar más estudios, por otra parte, para cuantificar el grado de conservación de la fauna de moluscos terrestres en la Sierra de Tamaulipas. Sin embargo, es

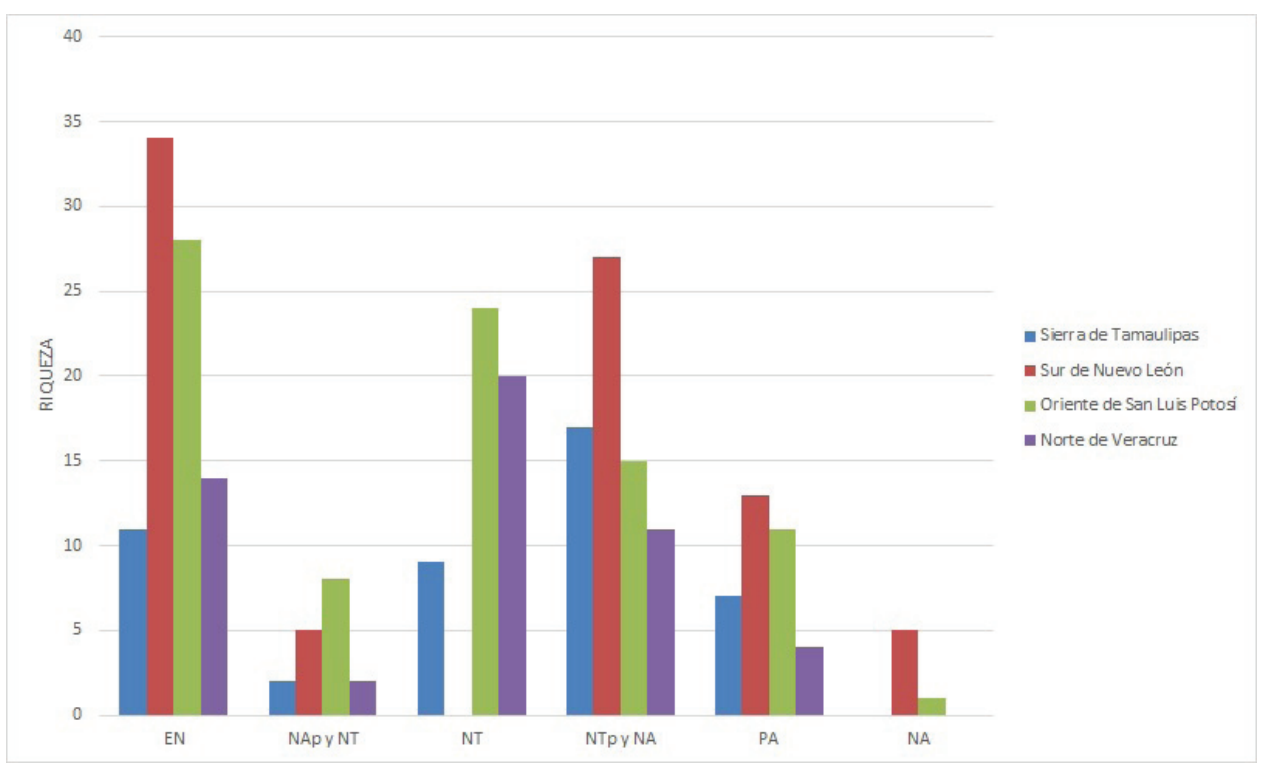

Figura 4. Comparativo de componentes zoogeográficos por número de especies de moluscos terrestres de la Sierra de Tamaulipas y otras áreas del noreste de México. Simbología del Cuadro 2. 


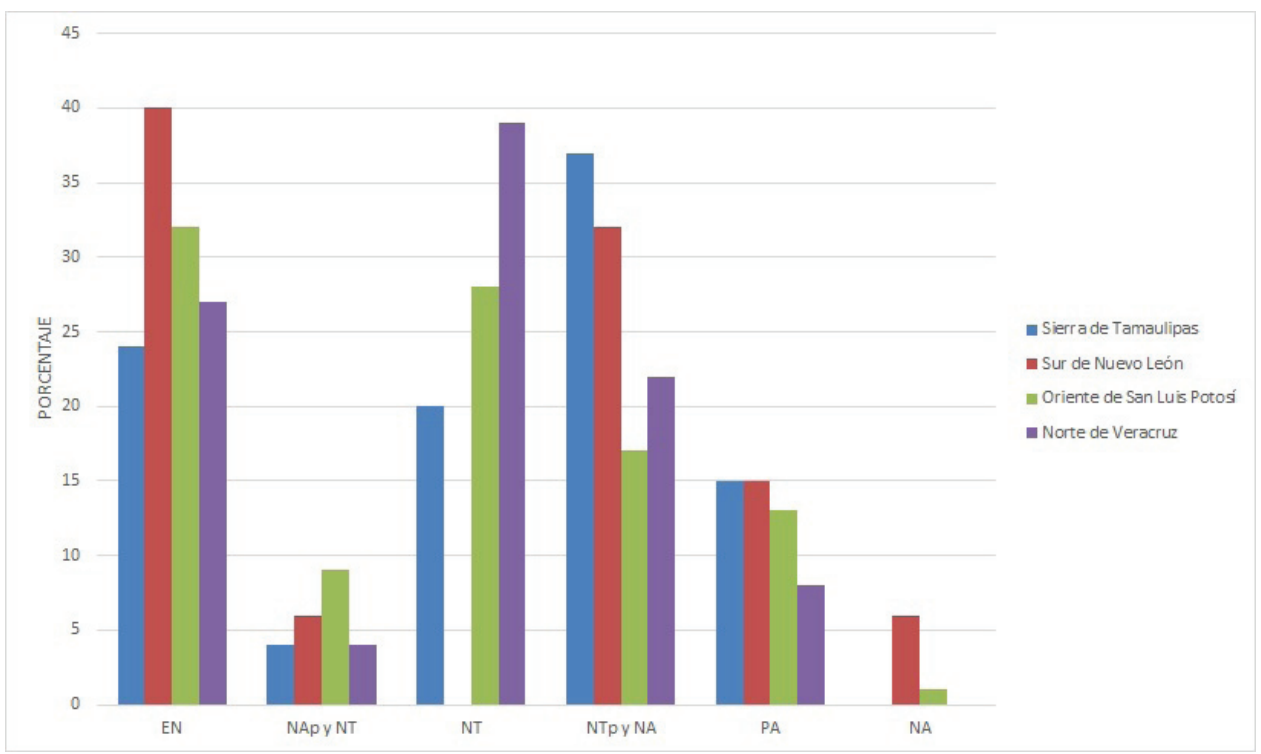

Figura 5. Comparativo porcentual de componentes zoogeográficos de la Sierra de Tamaulipas y otras áreas del noreste de México. Simbología del Cuadro 2.

posible aproximarse en este sentido. Áreas con vegetación original de bosque tropical subcaducifolio llegan a presentar 31 especies de gastrópodos terrestres y, en contraste, en las áreas adyacentes en donde la vegetación ha sido modificada y se han establecido áreas de cultivo se pueden registrar hasta 23 especies. La diferencia entre los dos tipos de vegetación oscila alrededor del 25.8\% (ocho especies).

El número de especies de gastrópodos que potencialmente pueden encontrarse en áreas de $9 \mathrm{~km}^{2}$ en el área de estudio fueron de 25 a 30 especies (Fig. 6), siendo más frecuente encontrar 28 especies. La mayor riqueza se encontró fuera del polígono de la Sierra de Tamaulipas, principalmente al norte de esta área, mientras que la menor riqueza se predice en el sureste. Las especies Ceres nelsoni y Streptostyla bartshii (conocidas para la Sierra

Cuadro 4. Familias de gastrópodos terrestres con endemismos y su porcentaje en relación al número total de especies por familia en la Sierra de Tamaulipas, México

\begin{tabular}{lcc}
\hline Familia & $\begin{array}{c}\text { Especies o subespecies } \\
\text { endémicas }\end{array}$ & $\begin{array}{c}\text { Porcentaje } \\
(\%)\end{array}$ \\
\hline Helicinidae & 1 & 20 \\
Zonitidae & 1 & 50 \\
Spiraxidae & 6 & 60 \\
Eucalodiidae & 1 & 100 \\
\hline Polygyridae & 2 & 33 \\
\hline
\end{tabular}

Madre Oriental) potencialmente pueden encontrarse en la Sierra de Tamaulipas, pero hasta ahora no han sido registradas. Por lo anterior es necesario incrementar la colecta en áreas que no han sido evaluadas para verificar este aspecto y establecer también, aún con mayor precisión, diversos aspectos de esta malacofauna.

AGRADECIMIENTOS. A la L.I. Anabel Gutiérrez Borbolla por su siempre paciente asistencia. Al Consejo Nacional de Ciencia y Tecnología por el apoyo económico. Al Dr. Fred G. Thompson por la literatura proporcionada y acceso a la Colección de Moluscos del Museo de Historia Natural de Florida, Universidad de Florida. Al M. C. Maximino Hernández por la elaboración de la Figura 1. A la Dra. Rosalía Guerrero Arenas, un revisor anónimo y los editores de Acta Zoológica Mexicana (Nueva Serie) cuyas correcciones y comentarios mejoraron sustancialmente este trabajo.

Cuadro 5. Índice biogeográfico por tipos de vegetación compartidos en la Sierra de Tamaulipas y Oriente de San Luis Potosí (OSLP).

\begin{tabular}{lcc}
\hline Tipo de vegetación & Sierra Tamaulipas & OSLP \\
\hline Bosque de encino & 4.3 & 4.70 \\
Bosque tropical & 4.3 & 4.55 \\
subcaducifolio & & \\
Pastizal & 2.75 & 4.40 \\
$\overline{\mathrm{x}}$ & 3.78 & 4.55 \\
\hline
\end{tabular}



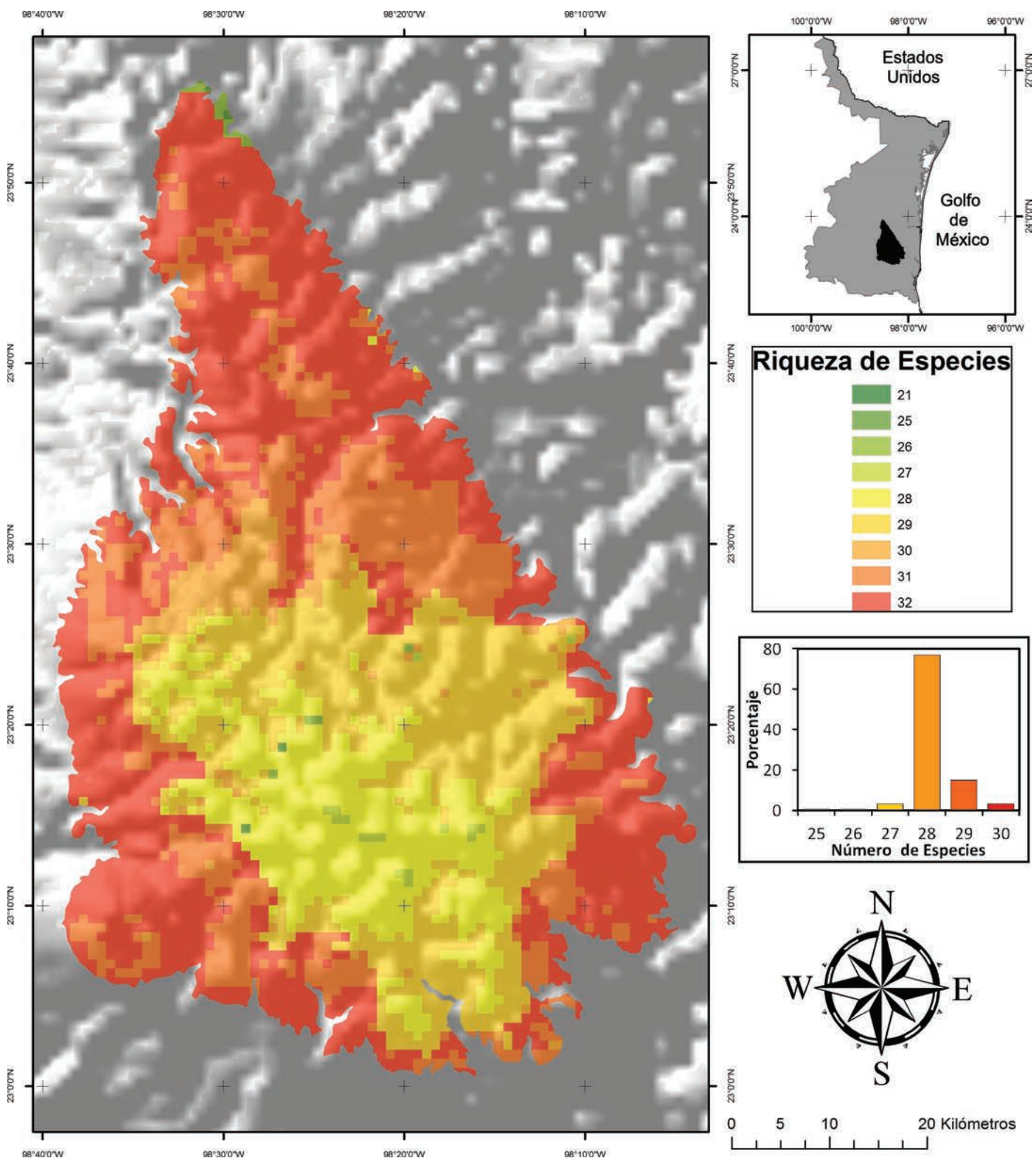

Figura 6. Mapa de la riqueza potencial de especies de moluscos terrestres en la Sierra de Tamaulipas (ver Apéndice 2). 


\section{LITERATURA CITADA}

Avendaño, M. J. 2004. Inventario de gasterópodos terrestres y dulceacuícolas del área focal de Ixcan, Chiapas. Instituto de Historia Natural y Ecología. Informe final SNIB-CONABIO. Proyecto No. Y015. México, D. F.

Avendaño, M. J., Carbot-Chanona, G. \& Naranjo-García, E. 2010. Moluscos gasterópodos terrestres y dulceacuícolas del área focal Ixcan, Chiapas, México. Lacandonia, 4, 29-36.

Bouchet, P. \& Rocroi, J.-P. 2005. Classification and nomenclature of gastropod families. Malacologia, 47, 1-397.

Cameron, R. A. A. \& Redfern, M. 1976. A sinopsis of the British land snails. Academic, Londres, Inglaterra.

Coney, C. C., Tarpley, W. A. \& Bohannan, R. 1981. A method of collecting minute land snails. Nautilus, 95, 43-44.

Correa-Sandoval, A. 1993. Caracoles terrestres (Mollusca: Gastropoda) de Santiago, Nuevo León, México. Revista de Biología Tropical, 41, 683-687.

Correa-Sandoval, A. 1999. Zoogeografía de los gastrópodos terrestres de la región oriental de San Luis Potosí, México. Revista de Biología Tropical, 47, 493-502.

Correa-Sandoval, A. 2000. Gastrópodos terrestres del norte de Veracruz, México. Acta Zoológica Mexicana (n.s.), 79, 1-9.

Correa-Sandoval, A. 2003. Gastropódos terrestres del noreste de México. Revista de Biología Tropical, 51, 507-522.

Correa-Sandoval, A. \& Salazar- Rodríguez, M. C. 2005. Gastrópodos terrestres del sur de Nuevo León, México. Acta Zoológica Mexicana (n.s.), 21, 51-61.

Correa-Sandoval, A. \& Rodríguez Castro, R. 2005. Gastrópodos terrestres. pp. 340-344. In: Sánchez, G., Reyes, P. \& Dirzo, R. (Eds.). Historia Natural de la Reserva de la Biosfera "El Cielo", Tamaulipas, México. Universidad Autónoma de Tamaulipas, Ciudad Victoria, México.

Correa- Sandoval, A., García-Cubas, A. \& Reguero, M. 1998. Gastrópodos terrestres de la región oriental de San Luis Potosí. México. Acta Zoológica Mexicana (n. s.), 73, 1-17.

Correa-Sandoval, A., Strenth, N. E. \& Salazar-Rodríguez, M. C. 2007. Zoogeografía de los gastrópodos terrestres del Sur de Nuevo León, México. Acta Zoológica Mexicana (n. s.), 23, 143-162.

Correa-Sandoval, A., Strenth, N. E., Rodríguez Castro, R. \& Horta-Vega, J. V. 2009. Análisis ecológico básico de los gastrópodos terrestres de la región oriental de San Luis Potosí, México. Acta Zoológica Mexicana (n.s.), 25, 105-122.

Correa-Sandoval, A., Martínez, V., Horta-Vega, J. V. \& Castro, I. 2012. Zoogeografía de los gastrópodos terrestres del Sur de Tamaulipas, México. Revista de Biología Tropical, 60, 317-331.

Elith, J., Graham, C. H., Anderson, R. P., Dudik, M., Ferrier, S., Guisan, A., Hijmans, R. J., Hueffmann, F., Leathwick, J. R., Lehmann, A., Li, J., Lohmann, L. G., Loiselle, B. A., Manion, G., Moritz, C., Nakamura, M., Nakazawa, Y., Overton, J. M., Peterson, A. T., Phillips, S. J., Richardson, K., Scachetti-Pereira, R., Schapire, R. E., Soberón, J., Williams, S., Wisz, M. S. \&
Zimmermann, N. E. 2006. Novel methods improve prediction of species of species' distributions from occurrence data. Ecography, 29, 129-151.

Guisan, A. \& Zimmermann, N. E. 2000. Predictive habitat distribution models in ecology. Ecological Modelling, 135, 147-186.

Hirzel, A. H. \& Lay, G. L. 2008. Habitat suitability modelling and niche theory. Journal of Applied Ecology, 45, 1372-1381.

Martínez-Ramírez, V. H. \& Rodríguez-Castro, R. 1997. Sistemática y aspectos ecológicos de los moluscos terrestres de los cañones (El Novillo, Calamaco y La Peregrina) al oeste de Ciudad Victoria, Tamaulipas, México. Tesis Profesional. Licenciatura en Biología. Instituto Tecnológico de Ciudad Victoria. 42 pp.

Pérez, A. M., Villaseca, J. C. \& Zione, N. 1996. Sinecología básica de moluscos terrestres en cuatro formaciones vegetales de Cuba. Revista de Biología Tropical, 44, 133-146.

Phillips, S. J., Anderson, R. P. \& Schapire, R. E. 2006. Maximum entropy modeling of species geographic distributions. Ecological Modelling, 190, 231-259

Pilsbry, H. A. 1920. Review of the Thysanophora plagioptycha group. Nautilus, 33, 93-96.

Pilsbry, H. A. \& Vanatta, E. G. 1936. Three Mexican Euglandina. Nautilus, 49, 97-98.

Rangel-Ruiz, L. \& Gamboa-Aguilar, J. 1998. Estudio taxonómico de moluscos terrestres y dulceacuícolas de la región maya de México I. Reserva de la Biosfera Pantanos de Centla. Universidad Juárez Autónoma de Tabasco. Informe final SNIB-CONABIO. Proyecto No. GO34. México, D. F.

Rangel-Ruiz, L. J., Gamboa-Aguilar, J. A. \& Alegría, F. R. 2004. Diversidad malacológica en la Región Maya. II. "Parque Estatal Agua Blanca”. Tabasco, México. Acta Zoológica Mexicana (n.s.), 20, 55-62.

Richards, C. L., Carstens, B. C. \& Knowles, L. 2007. Distribution modelling and statistical phylogeography: an integrative framework for generation and testing alternative biogeographical hypotheses. Journal of Biogeography, 34, 1833-1845.

Soberón, J. \& Peterson, A. T. 2005. Interpretation of models of fundamental ecological niches and species distributional areas. Biodiversity Informatics, 2, 1-10.

Solem, A., Emerson, W. K., Roth, B. \& Thompson, F. G. 1980. Standars for malacological collections. Curator, 24, 19-28.

Tattersfield, P., Waruj, C. M., Seddon, M. B. \& Kiringe, J. W. 2001. Land snails faunas of afromontane forest of Mount Kenya, Kenya: ecology, diversity and distribution patterns. Journal of Biogeography, 28, 843-861.

Thompson, F. G. (2011. Ann Annotated Checklist and Bibliography of the Land and Freshwater Snails of México and Central America Florida Museum of Natural History, University of Florida. Gainesville, Florida, U.S.A. Bulletin of the Florida Museum of Natural History, 50, 1-299.

Thompson, F. G. \& Correa- Sandoval, A. 1994. Land snails of the genus Coelocentrum from northeastern Mexico. Bulletin of the Florida Museum of Natural History, 36, 141-173. 


\section{APÉNDICE 1}

\section{Localidades visitadas en la Sierra de Tamaulipas}

\begin{tabular}{|c|c|c|c|}
\hline & LOCALIDAD & \multicolumn{2}{|c|}{$\begin{array}{l}\text { COORDENADAS } \\
\text { GEOGRÁFICAS }\end{array}$} \\
\hline 1 & Km 52. Carretera Victoria - Soto La Marina después de Villa de Casas & $23^{\circ} 39^{\prime} 83^{\prime \prime}$ & $98^{\circ} 39^{\prime} 42^{\prime \prime}$ \\
\hline 2 & Km 64 Carretera Victoria - Soto La Marina & $23^{\circ} 34^{\prime} 99^{\prime \prime}$ & $98^{\circ} 36^{\prime} 15^{\prime \prime}$ \\
\hline 3 & km 68 Carretera Victoria - Soto La Marina & $23^{\circ} 34^{\prime} 42^{\prime \prime}$ & $98^{\circ} 33^{\prime} 29^{\prime \prime}$ \\
\hline 4 & Km 64.5 Carretera Victoria - Soto La Marina, después de El Pirulí & $23^{\circ} 34^{\prime} 37^{\prime \prime}$ & $98^{\circ} 31^{\prime} 29^{\prime \prime}$ \\
\hline 5 & Km 74 Carretera Victoria - Soto La Marina, antes de El Sabinito & $23^{\circ} 34^{\prime} 30^{\prime \prime}$ & $98^{\circ} 30^{\prime} 81^{\prime \prime}$ \\
\hline 6 & Km 115 Carretera Victoria - Soto La Marina & $23^{\circ} 43^{\prime} 56^{\prime \prime}$ & $98^{\circ} 13^{\prime} 62^{\prime \prime}$ \\
\hline 7 & Km 104 Carretera Victoria - Soto La Marina & $23^{\circ} 41^{\prime} 23^{\prime \prime}$ & $98^{\circ} 17^{\prime} 56^{\prime \prime}$ \\
\hline 8 & $\begin{array}{l}\text { Rancho La Palma, Carretera Victoria - Soto La Marina, lado izquierdo } \\
\text { de la carretera }\end{array}$ & $23^{\circ} 34^{\prime} 36^{\prime \prime}$ & $98^{\circ} 29^{\prime} 77^{\prime \prime}$ \\
\hline 9 & $\begin{array}{l}\text { Rancho La Palma Carretera Victoria - Soto La Marina, lado derecho de } \\
\text { la carretera }\end{array}$ & $23^{\circ} 34^{\prime} 30^{\prime \prime}$ & $98^{\circ} 29^{\prime} 43^{\prime \prime}$ \\
\hline 10 & Carretera La Pesca - Soto La Marina, después de Vista Hermosa & $23^{\circ} 48^{\prime} 98^{\prime \prime}$ & $97^{\circ} 55^{\prime} 52^{\prime \prime}$ \\
\hline 11 & Carretera La Pesca - Soto La Marina, antes de la desviación al Chapote & $23^{\circ} 44^{\prime} 94^{\prime \prime}$ & $97^{\circ} 55^{\prime} 57^{\prime \prime}$ \\
\hline $\begin{array}{l}12 \\
13\end{array}$ & $\begin{array}{l}\text { Cerro Gordo, Ej. La Peña, mpio. de Aldama Tam. } \\
\text { Carretera Victoria - Soto La Marina, Ej. El Nogalito }\end{array}$ & $23^{\circ} 31^{\prime} 00^{\prime \prime}$ & $98^{\circ} 32^{\prime} 03^{\prime \prime}$ \\
\hline 14 & Rancho El Carrizo, km 25 Carretera Victoria - Soto La Marina & $23^{\circ} 47^{\prime} 51^{\prime \prime}$ & $97^{\circ} 59^{\prime} 39^{\prime \prime}$ \\
\hline 15 & Carretera San José de las Rusias - Tepehuajes, km 10 & $23^{\circ} 32^{\prime} 37^{\prime \prime}$ & $97^{\circ} 55^{\prime} 51^{\prime \prime}$ \\
\hline 16 & Ej. Vista Hermosa, carretera Soto La Marina - La Pesca & $23^{\circ} 48^{\prime \prime} 31^{\prime \prime}$ & $97^{\circ} 56^{\prime} 33^{\prime \prime}$ \\
\hline 17 & Carretera La Pesca - Soto La Marina después del entronque al Capote & $23^{\circ} 47^{\prime} 51^{\prime \prime}$ & $98^{\circ} 04^{\prime} 07^{\prime \prime}$ \\
\hline 18 & Soto la Marina, Tamaulipas & $23^{\circ} 44^{\prime} 54^{\prime \prime}$ & $98^{\circ} 12^{\prime} 45^{\prime \prime}$ \\
\hline 19 & $\begin{array}{l}\text { Ej. El Sabinito km } 97 \text { Carretera Victoria - Soto La Marina, derecha de } \\
\text { la carretera }\end{array}$ & $23^{\circ} 35^{\prime} 34^{\prime \prime}$ & $98^{\circ} 21^{\prime} 22^{\prime \prime}$ \\
\hline 20 & Rancho los Ebanos, Carretera Tepehuajes - San José de las Rusias & $23^{\circ} 30^{\prime} 00^{\prime \prime}$ & $97^{\circ} 49^{\prime} 18^{\prime \prime}$ \\
\hline 21 & Después del vado El Moro, carr. Victoria-Soto La Marina, km 69 & $23^{\circ} 35^{\prime} 14^{\prime \prime}$ & $98^{\circ} 37^{\prime} 34^{\prime \prime}$ \\
\hline 22 & Ejido La Lajilla, al sureste de Casas & $23^{\circ} 38^{\prime} 15^{\prime}$, & $98^{\circ} 33^{\prime} 26^{\prime}$ \\
\hline 23 & Villa de Casas & $23^{\circ} 42^{\prime} 37^{\prime}$ & $98^{\circ} 43^{\prime} 47^{\prime}$ \\
\hline 24 & $\begin{array}{l}\text { Rancho La Palma, km 80, después de El Pirulí, desviación al Ejido } \\
\text { Felipe Ángeles }\end{array}$ & $23^{\circ} 31^{\prime} 57^{\prime \prime}$ & $98^{\circ} 31^{\prime} 22^{\prime}$, \\
\hline 25 & Rancho La Palma, km 77, carr. Victoria-Soto La Marina & $23^{\circ} 34^{\prime} 37^{\prime}$ & $98^{\circ} 24^{\prime} 07^{\prime \prime}$ \\
\hline 26 & Carr. Victoria-Soto La Marina, km. 23 & $23^{\circ} 42^{\prime} 17^{\prime}$ & $98^{\circ} 55^{\prime} 51^{\prime \prime}$ \\
\hline 27 & Unidad Deportiva de Soto La Marina & $23^{\circ} 45^{\prime} 14^{\prime}$ & $98^{\circ} 13^{\prime} 26^{\prime}$, \\
\hline 28 & Carr. La Pesca-Soto La Marina (después de entronque El Capote) & $23^{\circ} 47^{\prime} 51^{\prime}$ & $98^{\circ} 04^{\prime} 07^{\prime \prime}$ \\
\hline 29 & Interior Rancho Los Ébanos, Soto La Marina & $23^{\circ} 26^{\prime} 13^{\prime \prime}$ & $97^{\circ} 47^{\prime} 34^{\prime \prime}$ \\
\hline 30 & Ejido El Porvenir (arroyo), Soto La Marina & $23^{\circ} 31^{\prime} 37^{\prime}$ & $97^{\circ} 53^{\prime} 26^{\prime}$, \\
\hline
\end{tabular}




\section{APÉNDICE 2}

Especies de moluscos terrestres de la Sierra de Tamaulipas, afinidades biogeográficas y áreas de distribución local.* Especies a las que se les generó el modelo de distribución geográfica potencial.

\begin{tabular}{|c|c|c|c|}
\hline Especies & Afinidad & Localidades & $\begin{array}{c}\text { Total } \\
\text { localidades }\end{array}$ \\
\hline \multicolumn{4}{|l|}{ FAMILIA HELICINIDAE } \\
\hline Helicina chrysocheila chrisocheyla (Binney, 1851)* & NTp y NA & $\begin{array}{l}1,2,3,4,5,8,9,10,14,15,17,19,20,21,23,24 \\
28,29,30 .\end{array}$ & 20 \\
\hline Helicina orbiculata orbiculata (Say, 1818)* & NAp y NT & $1,2,10,11,15,22$ & 6 \\
\hline Helicina zephyrina zephyrina (Duclos, 1833)* & NT & $10,11,14,15,17,19,20,24,25,28,29$. & 11 \\
\hline Schasicheyla fragilis Pilsbry 1899 & EN & $1,3,4,10,29$. & 5 \\
\hline \multicolumn{4}{|l|}{ FAMILIA VERONICELLIDAE } \\
\hline Leidyula moreleti (Fischer, 1871)* & NT & 15, 21. & 2 \\
\hline \multicolumn{4}{|l|}{ FAMILIA SUCCINEIDAE } \\
\hline Succinea luteola luteola (Gould, 1848)* & PA & $1,7,10,11,20,29,30$ & 7 \\
\hline \multicolumn{4}{|l|}{ FAMILIA STROBILOPSIDAE } \\
\hline Strobilops hubbardi (Brown, 1861) & PA & $8,10,11$ & 3 \\
\hline \multicolumn{4}{|l|}{ FAMILIA VERTIGINIDAE } \\
\hline Gastrocopta pellucida hordeacella (Pilsbry, 1890)* & PA & $1,2,3,4,5,7,8,9,10,11,14,21$ & 12 \\
\hline Pupisoma dioscoricola insigne (Pilsbry 1920)* & NTp y NA & $1,3,4,8,9,10,11,13,14,21$ & 10 \\
\hline \multicolumn{4}{|l|}{ FAMILIA ORTHALICIDAE } \\
\hline Drymaeus emeus (Say, 1829)* & NTp y NA & $1,2,3,8,10,11,14,19,20,21,24$ & 11 \\
\hline Drymaeus multilineatus (Say, 1825)* & NTp y NA & $1,2,10,11,14,15,20,22,23,29$ & 10 \\
\hline Drymaeus sulfureus (Pfeiffer, 1856) & NT & $3,14,15,20,29$ & 5 \\
\hline Orthalicus princeps princeps (Broderip, 1833) & NT & 15, 20, 29. & 3 \\
\hline Rabdotus alternatus alternatus (Say, 1830)* & NT & 1, 2, 10, 22, 27 & 5 \\
\hline Rabdotus dealbatus dealbatus (Say, 1821)* & PA & $15,17,18,22,26,28,30$ & 7 \\
\hline \multicolumn{4}{|l|}{ FAMILIA EUCALODIIDAE } \\
\hline $\begin{array}{l}\text { Coelocentrum torosum (Thompson \& Correa-Sandoval, } \\
\text { 1994) }\end{array}$ & EN & 1, 3, 4, 9, 10, 14, 19, 21 & 8 \\
\hline \multicolumn{4}{|l|}{ FAMILIA UROCOPTIDAE } \\
\hline Microceramus mexicanus (Martens, 1897)* & NTp y NA & $3,8,24,30$ & 4 \\
\hline \multicolumn{4}{|l|}{ FAMILIA FERUSSACIDAE } \\
\hline Cecilioides consobrinus primus (De Folin, 1870)* & NT & $1,2,3,5,8,9,10,11,15$ & 9 \\
\hline \multicolumn{4}{|l|}{ FAMILIA SUBULINIDAE } \\
\hline Leptopeas micra micra (Orbigny, 1835) & NTp y NA & $1,2,8,9,10,11$ & 6 \\
\hline Lamellaxis tamaulipensis (Pilsbry, 1903)* & NT & $3,4,8,15,19,30$ & 6 \\
\hline Euglandina jacksoni Pilsbry \& Vannata, 1936 & EN & 21 & 1 \\
\hline Euglandina sp. nov. & EN & 24 & 1 \\
\hline Euglandina texasiana texasiana (Pfeiffer, 1856).* & NTp y NA & $1,2,3,4,8,9,14,19,20,21,24,25,29,30$ & 14 \\
\hline Guillarmodia dalli (Pilsbry, 1899)* & EN & $4,8,24$ & 3 \\
\hline G. potosiana tamaulipensis (Pilsbry, 1908)* & NTp y NA & 4,19, 21 & 3 \\
\hline G. victoriana (Pilsbry, 1903)* & EN & $3,4,8,10,11,24,30$ & 7 \\
\hline Salasiella hinkleyi (Pilsbry, 1920) & EN & $2,3,4$ & 3 \\
\hline
\end{tabular}




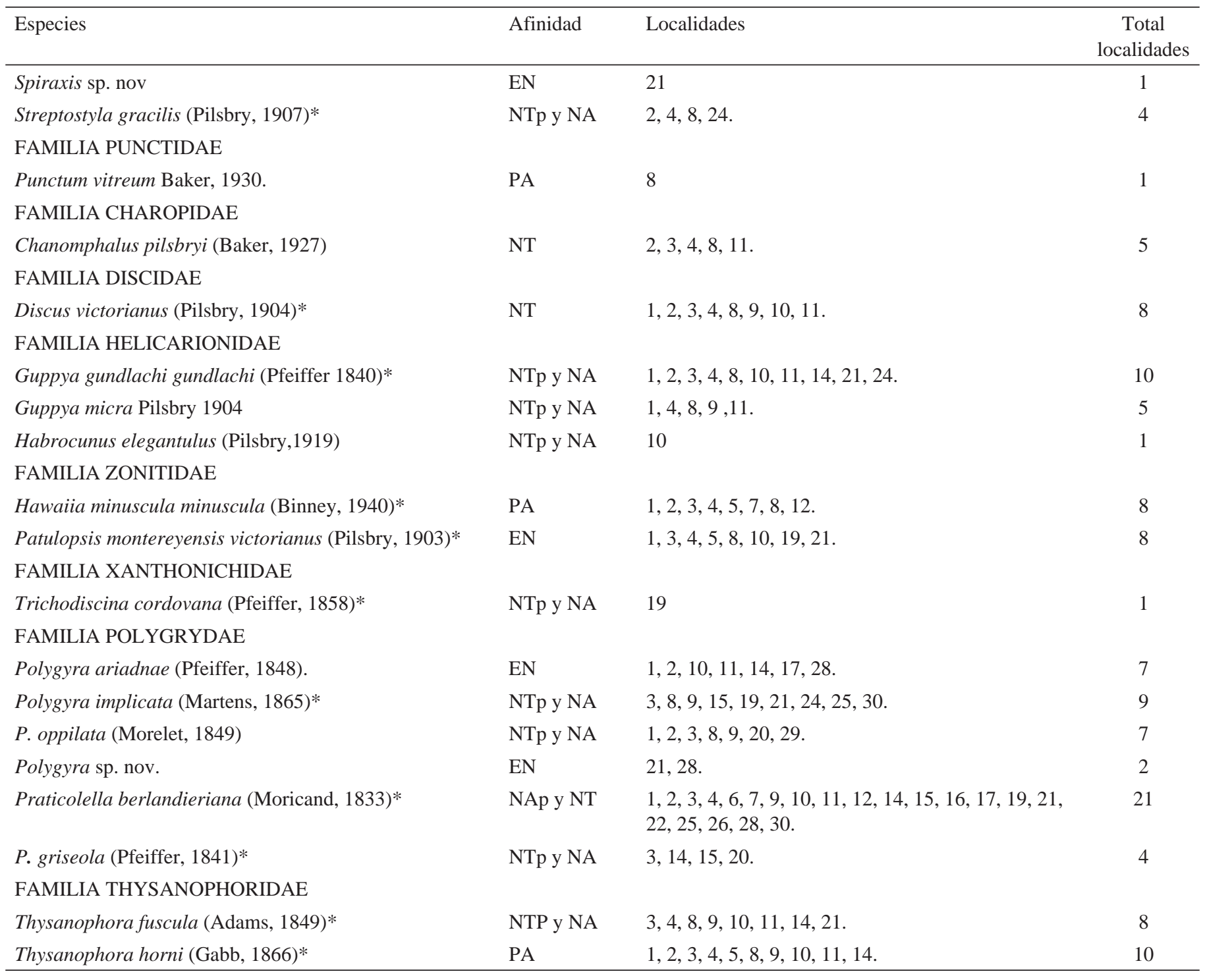

